\title{
A 45-year-old woman with a pre-sacral mass lesion: diagnosis and discussion
}

\author{
Inderjeet Nagra • Alistair J. Stirling • Steven L. J. James
}

Published online: 26 November 2009

(C) ISS 2009

Keywords Endometriosis · Foraminal expansion $\cdot$ Sacrum

\section{Diagnosis}

Endometriosis presenting as a pre-sacral mass lesion causing sacral foraminal expansion and $\mathrm{S} 1$ radiculopathy.

\section{Discussion}

The MR examination demonstrates a $6 \times 2.5-\mathrm{cm}$ pre-sacral mass lesion that is extending into and expanding the left $\mathrm{S} 1$ exit foramen. This mass extends into the epidural fat, displacing and partially surrounding the $\mathrm{S} 1$ nerve, but it did not extend distally to affect the exiting S2 or S3 nerves. The lesion shows a heterogeneous signal pattern on T1- and T2weighted sequences with focal areas of high signal intensity on the T1-weighted sequences indicative of small areas of haemorrhage. The lesion is predominantly hyperintense to muscle on T2 weighting and following contrast medium administration, and a relatively uniform enhancement pattern is identified. Trans-sacral biopsy by an approach

The case presentation can be found at doi:10.1007/s00256-009-0831-6

I. Nagra $\cdot$ S. L. J. James $(\bowtie)$

Department of Radiology,

The Royal Orthopaedic Hospital NHS Foundation Trust, Birmingham B31 2AP, UK

e-mail: steven.james@roh.nhs.uk

\section{A. J. Stirling}

Department of Spinal Surgery,

The Royal Orthopaedic Hospital NHS Foundation Trust, Birmingham B31 2AP, UK lateral to the exit foramen was performed to exclude recurrent rectal carcinoma. The biopsy material demonstrated endometriosis characterised by endometrial glands surrounded by endometrial stroma with strong positivity for oestrogen and progesterone. As a result, the patient was commenced on hormonal treatment and the neurological symptoms improved.

Although endometriosis is a common condition affecting women of reproductive age, it may rarely affect both the central and peripheral nervous systems. The exact prevalence is unknown, but a greater number of cases affecting the peripheral nervous system have been reported, the commonest being the sciatic nerve [1]. Presentation is typically with cyclical pain in a sciatic distribution with sensory loss in the S1 dermatome and motor weakness. Symptoms may result from either direct compression or fibrosis of the nerve. The symptoms are usually progressive unless treated and prolonged compression can lead to irreversible damage.

A number of theories have been postulated to explain the extra-uterine spread of endometriosis [2]. In the ovary, the commonest site of endometriosis outside of the uterus, lymphatic drainage from the uterus has been suggested to be the cause [3]. Distant sites of extra-pelvic endometriosis, such as the thorax or central nervous system, have been attributed to haematological transport, but coeloemic tissue metaplasia has also been postulated, as endometriosis can occur in patients who have never menstruated or even in post-menopausal women [4]. This has been refuted by other workers who have found asymmetric sciatic nerve endometriosis and diaphragmatic disease more commonly on the right side, supporting the menstrual reflux theory because of the transport of endometrial cells in the cycle of peritoneal fluid redistribution [5]. In the pelvis, the left 
Table 1 Benign and malignant conditions causing spinal foraminal widening

\begin{tabular}{ll}
\hline Benign conditions & Malignant conditions \\
\hline Benign peripheral nerve sheath tumour & Malignant peripheral nerve sheath tumour \\
Tarlov cyst & Metastases/lymphoma \\
Spinal hydatid disease & Chordoma \\
Tuberculous or pyogenic spondylitis & Solitary plasmacytoma \\
\hline
\end{tabular}

sciatic and lumbosacral plexus is protected by interposition of the sigmoid colon and in the right hypochondrium, the falciform ligament acts as a site for implantation [5]. In this case, it seems likely that the lesion originated in the pre-sacral space and extended into the epidural fat, subsequently expanding the S1 foramen.

The inherent soft tissue contrast of MR imaging makes this the method of choice for evaluating endometriosis $[1,6]$. Lesions are typically of high signal intensity on T1- and T2-weighted sequences with a surrounding low intensity rim due to the presence of a fibrous capsule [1, 6]. Fat-suppressed and STIR sequences make endometriotic lesions more conspicuous. The areas of central high signal intensity on T1-weighted sequences are caused by blood degradation products of different ages and the paramagnetic effects of methaemoglobin. Variable signal intensity was seen in our case because of the quantity and age of the haemorrhage and the proportion of endometrial cells and stroma. Endometriotic lesions do not usually enhance, although mild enhancement can occur, as was identified in this case.

The differential diagnosis for mass lesions causing neural foraminal widening and nerve root symptoms is wide and includes both benign and malignant conditions (see Table 1).

The commonest differential diagnosis is a peripheral nerve sheath tumour (PNST), which can be divided into benign (neurofibroma or schwannoma) and malignant categories. They are soft tissue tumours arising from the spinal nerve root and may have a dumbbell shape on MR imaging. They are slow-growing and plexiform neurofibromas can involve a long segment of nerve. They are usually of low signal intensity on T1- and of high signal intensity on T2-weighted sequences [7]. The signal characteristics may be difficult to distinguish from endometriosis as lesions can appear heterogeneous if internal degeneration or necrosis is present, as is commonly identified in schwannomas. Malignant PNSTs grow more rapidly, show central necrosis and have an ill-defined border [7]. Other benign differentials to be considered are tuberculosis, more commonly occurring in the cervical or thoracic spine. Uncommonly, hydatid cysts present with a lesion affecting a vertebral body as a result of a portovertebral shunt. They tend to be multicystic, cause bone destruction, but typically preserve the disc space and do not involve the spinal cord [8]. Chordomas are rare, aggressive malignant tumours arising from ectopic notochord remnants, especially in the sacro-coccygeal or spheno-occipital-clival regions. On MR imaging, they display low to intermediate signal intensity on T1- and very high signal intensity on T2-weighted sequences. Chordomas enhance heterogeneously and cause local bony destruction. Haemorrhagic necrosis can also occur within the tumour leading to areas of high signal on T1 [9].

Early diagnosis of endometriosis is important as prolonged compression and fibrosis of nerves can lead to irreversible damage. Treatment options depend on whether fertility is to be preserved, as preferential treatment would then be directed towards pharmacological suppression of ovarian hormones rather than surgical resection of the endometriotic lesions. In selected patients in whom medical therapy has failed, excisional surgery to decompress the nerve can be considered. In older patients or in resistant cases, a hysterectomy and oophorectomy can be considered combined with hormonal therapy.

\section{References}

1. Yekeler E, Kumbasar B, Tunaci A, Barman A, Bengisu E, et al. Cyclical sciatica caused by infiltrative endometriosis: MRI findings. Skeletal Radiol. 2004;33:165-8.

2. Mannan K, Altaf F, Maniar S, Tirabosco R, Sinisi M, Carlstedt T. Cyclical sciatica: endometriosis of the sciatic nerve. J Bone Jt Surg Br. 2008;90(1):98-101.

3. Ueki M. Histologic study of endometriosis and examination of lymphatic drainage in and from the uterus. Am J Obstet Gynecol. 1991;165(1):201-9.

4. Suginami H. A reappraisal of the coelomic metaplasia theory by reviewing endometriosis occurring in unusual sites and instances. Am J Obstet Gynecol. 1991;165:214-18.

5. Vercellini P, Abbiati A, Viganò P, Somigliana ED, Daguati R, Meroni F, et al. Asymmetry in distribution of diaphragmatic endometriotic lesions: evidence in favour of the menstrual reflux theory. Hum Reprod. 2007;22:2359-67.

6. Moeser P, Donofrio PD, Karstaedt N, Bechtold R, Greiss FC Jr. MRI findings of sciatic endometriosis. Clin Imaging. 1990;14:64-6.

7. Lin J, Martel W. Cross-sectional imaging of peripheral nerve sheath tumours: characteristics on CT, MR and US. Am J Roentgenol. 2001;176:75-82.

8. Zibis AH, Markonis A, Karantanas AH. Unusual causes of spinal foraminal widening. Eur Radiol. 2000;10:144-8.

9. Kivrak AS, Koc O, Emlik D, Kiresi D, Odev K, Kalkan E. Differential diagnosis of dumbbell lesions associated with spinal neural foraminal widening: imaging features. Eur J Radiol. 2009;71:29-41. 